\title{
EmergenciApp: aplicación personalizada para el direccionamiento de la atención de emergencias
}

\section{EmergenciApp: personalized app for directing emergency care}

\author{
Miguel Ángel Páez-Ramos ${ }^{1}$, Andrés David Mariño-Sánchez ${ }^{1}$, Alejandro Mayorga-Cortés ${ }^{1}$, \\ Ángela Carrillo-Ramos ${ }^{1}$ \\ ${ }^{1}$ Pontificia Universidad Javeriana, Bogotá D.C., Colombia \\ miguelpaez@javeriana.edu.co,andres.marino@javeriana.edu.co,alejandromayorga@javeriana.edu.co, \\ angela.carrillo@javeriana.edu.co
}

(Recibido: 16 octubre 2020; aceptado: 19 abril 2021; Publicado en Internet: 1 junio 2021)

\begin{abstract}
Resumen. El bajo conocimiento por parte de las personas sobre las entidades que les pueden atender en situaciones de emergencia causa que éstas acudan ante el ente equivocado, y, por ende, no puedan ser atendidas correctamente. Esta problemática puede terminar afectando la integridad tanto física como mental de aquellos cuya situación de emergencia no termina siendo resuelta de forma adecuada. Por esta razón se propone EmergenciApp, una solución que aborde esta problemática sobre a quién y dónde acudir durante estas eventualidades. Si bien se plantea esta propuesta como capaz de atender cualquier tipo de emergencia, el presente artículo tiene como foco principal las emergencias de tipo médico, particularmente el re-direccionamiento hacia Instituciones Prestadoras de Salud (IPS), así como un sistema de notificación a seres queridos sobre emergencias que tenga el usuario.
\end{abstract}

Palabras clave: Emergencia, Re-direccionamiento, Personalización, Institución prestadora de salud, Pre-diagnóstico.

\begin{abstract}
The lack of knowledge of the people about the entities that can care for them in emergencies causes them to come to the wrong organization. Therefore, these organizations could not help people. This problem may end up affecting the physical and mental integrity of those whose emergency does not end up being resolved appropriately. We propose EmergenciApp, a solution that solves this problem about where and who can help the users during these events. While this system is conceptualized as capable of dealing with any emergency, this paper focuses on medical emergencies, particularly the redirection to medical centers and a system of notification to user's loved people about emergencies that the user has.
\end{abstract}

Keywords: Emergency, Redirect, Personalization, Health provider institutions, Pre-diagnosis.

Tipo de artículo: Artículo de investigación.

\section{Introducción}

El cuidado de la integridad física de las personas es algo que ha sido imprescindible desde el inicio de los tiempos, ya sea mediante el tratamiento médico, o con la atención y prevención de situaciones que ponen en peligro la vida de los individuos, por lo que este aspecto siempre ha sido objetivo a ser mejorado. Sin embargo, a día de hoy, siguen ocurriendo situaciones donde las personas no logran llegar a una entidad que sea capaz de ayudarles en estas inesperadas situaciones a razón de varios factores entre los cuales está el desconocimiento sobre a quién acudir durante estas situaciones.

Esta problemática causó que en la ciudad de Bogotá fueran reportadas más de 500.000 emergencias durante el año 2018 (Unidad Nacional para la Gestión del Riesgo de Desastres, 2019), de las cuales 89 acabaron en el denominado "paseo de la muerte" (Caracol Radio, 2018). Cabe mencionar que existen personas que acuden a una Institución Prestadora de Salud (IPS), y terminan perdiendo la vida al ser redireccionadas a otra, debido a que la persona o no cumple con los convenios médicos de la primera, o ésta no cuenta con los especialistas para tratar la afección/problema de salud del paciente.

Si bien para estos casos existe la línea de atención a emergencias y seguridad de Bogotá (Línea de emergencias de Bogotá, 2007), la cual ayuda a las personas durante estas situaciones enviando ambulancias o profesionales para el apoyo, hay desconocimiento y mal uso de la misma línea por parte de los ciudadanos 
(El Espectador, 2018), lo que causa que aquellos que, si necesitan ayuda, la obtengan con demoras e, incluso, de manera errónea. Es por esto que se propone EmergenciApp, una aplicación que pueda brindar a los ciudadanos información personalizada sobre a quién acudir, a qué números llamar, y cómo llegar a dichas entidades encargadas de resolver emergencias.

La finalidad del presente artículo es presentar una aplicación que aborde esta problemática y, además, brinde un valor agregado. Para lograr esto, se expondrán los conceptos básicos que se tratarán a lo largo del documento. Seguido, se enunciarán los trabajos relacionados encontrados con sus respectivas ventajas y desventajas. Luego, se describirá la solución propuesta con un conjunto de servicios a ofrecer y un modelo de usuario que servirá para que dichos servicios se adapten lo mejor posible a éste. Adicionalmente, se presentará el diseño e implementación de la aplicación en un prototipo funcional, con sus correspondientes pruebas y validaciones con usuarios reales. Por último, se presentarán las conclusiones y trabajo futuro.

\section{Conceptos de base}

En esta sección, se revisarán conceptos de base para entender la problemática y solución planteada. Primero, se expondrá la problemática a tratar: las emergencias que corresponden a situaciones fuera de control para el usuario que son de carácter urgente y donde puede estar en riesgo la vida de las personas involucradas. Dichas emergencias, se segmentan en cuatro partes importantes: la primera, relacionada con el área de salud, la segunda, corresponde a casos de emergencias policiales, tercero, emergencias de bomberos; finalmente, percances domésticos. Sin embargo, para el presente artículo sólo se tratarán las de salud, las cuales están relacionadas con cualquier situación donde la persona requiera atención médica por una eventualidad tal como accidentes que involucren heridos o agravamientos de ciertos padecimientos de forma inesperada, como, por ejemplo, un infarto.

Ahora, lo que se busca es brindar información al usuario sobre las entidades que le puedan ayudar, tal como datos de contacto y ubicación de las mismas. Es por esto que se realizó la siguiente pregunta: ¿Qué novedad se puede traer a un sistema de emergencias? La personalización, la cual consiste en adaptar un servicio o producto según características y gustos de un usuario particular. Mediante ésta, se puede mostrar la ubicación de todas estas entidades relacionadas con la salud, considerando ciertos rasgos del usuario. Por lo anterior, se tendrá en cuenta datos importantes, como lo son los seguros médicos de la persona, pues el sujeto podría dar prioridad a los hospitales o farmacias según los convenios con su medicina pre-pagada, por ejemplo. También, existe una novedad que se podría aportar a un sistema de apoyo en situaciones de emergencia, la cual consiste en notificar a los seres queridos, puesto que, con base en un evento inesperado sobre el miembro de una familia, sería ideal que los demás familiares de la persona en apuros estén informados de una manera automática sobre el suceso mediante un mensaje de texto (SMS) o correo electrónico, por ejemplo, guardando la confidencialidad de los datos del paciente.

Por último, se definen algunos términos recurrentes: las Entidades Prestadoras de Salud (EPS), y las Instituciones Prestadoras de Salud (IPS), las cuales son centros médicos que tienen convenio con la EPS particular de un usuario. Estos dos conceptos son de suma importancia para el proyecto pues se le brindará al usuario información acorde con sus datos y emergencias presentadas.

\section{Trabajos relacionados}

Existen trabajos relacionados que están apoyados en las tecnologías de la información y comunicaciones (TIC), que fueron agrupados según su funcionalidad principal de la siguiente forma: apoyar la toma de decisiones por parte de un médico, como MD+CAL (MDCalc, 2021) y Gyant (GYANT, 2021); proveer líneas de comunicación con centros de atención de emergencias, como Emergencia 911 (Ministerio del Interior, 2016), Línea de emergencia de Bogotá (Línea de emergencias de Bogotá, 2007), Help me -SOS International (International SOS, 2021), SOS Emergencias (S.O.S Emergencias, 2020), My112 (Comunidad de Madrid, 2015), y Safe 365 (Safe365, 2021); alojamiento de un perfil médico, como Medical ID (Medical ID, 2021); proveer información de primeros auxilios en caso de emergencia, como Primeros Auxilios Fáciles (App Primeros Auxilios, 2014); e informar a los servicios de pólizas con entidades aseguradoras, como Previsora Seguros (La Previsora S.A., 2020), Seguros Sura (Sura, 2017), y Seguros Bolívar (Seguros Bolívar, 2020). En la Tabla 1 se puede visualizar la comparación entre los diversos 
trabajos teniendo en cuenta criterios de personalización, de tecnologías de la información y de interacción por parte del usuario. Para cada criterio se muestran los trabajos que cumplen con el mismo.

Tabla 1. Trabajos relacionados

\begin{tabular}{|c|c|c|}
\hline & Criterio & Trabajos relacionados \\
\hline \multirow[t]{2}{*}{ TIC } & Aplicación móvil & $\begin{array}{l}\text { (App Primeros Auxilios, 2014; Comunidad de Madrid, 2015; } \\
\text { GYANT, 2021; International SOS, 2021; La Previsora S.A., } \\
\text { 2020; MDCalc, 2021; Medical ID, 2021; Ministerio del } \\
\text { Interior, 2016; S.O.S Emergencias, 2020; Safe365, 2021; } \\
\text { Seguros Bolívar, 2020; Sura, 2017). }\end{array}$ \\
\hline & Cuenta con acceso gratuito & $\begin{array}{l}\text { (Comunidad de Madrid, 2015; GYANT, 2021; International } \\
\text { SOS, 2021; Línea de emergencias de Bogotá, 2007; MDCalc, } \\
\text { 2021; Medical ID, 2021; Ministerio del Interior, 2016; S.O.S } \\
\text { Emergencias, 2020; Safe365, 2021). }\end{array}$ \\
\hline \multirow[t]{9}{*}{ Personalización } & Alergias & (Medical ID, 2021). \\
\hline & $\begin{array}{l}\text { Medicamentos de consumo } \\
\text { regular }\end{array}$ & (Medical ID, 2021). \\
\hline & $\begin{array}{l}\text { Entidad prestadora de servicio } \\
\text { médico }\end{array}$ & (Sura, 2017). \\
\hline & Póliza de seguro & (La Previsora S.A., 2020; Seguros Bolívar, 2020; Sura, 2017). \\
\hline & Avatar & (GYANT, 2021; Medical ID, 2021; Safe365, 2021). \\
\hline & Edad & $\begin{array}{l}\text { (GYANT, 2021; MDCalc, 2021; Medical ID, 2021; Safe365, } \\
\text { 2021; Sura, 2017). }\end{array}$ \\
\hline & Género & $\begin{array}{l}\text { (MDCalc, 2021; Medical ID, 2021; Safe365, 2021; Sura, } \\
\text { 2017). }\end{array}$ \\
\hline & Peso & $\begin{array}{l}\text { (GYANT, 2021; MDCalc, 2021; Medical ID, 2021; Sura, } \\
\text { 2017). }\end{array}$ \\
\hline & Contactos de emergencia & $\begin{array}{l}\text { (Comunidad de Madrid, 2015; La Previsora S.A., 2020; } \\
\text { Medical ID, 2021; Safe365, 2021; Seguros Bolívar, 2020). }\end{array}$ \\
\hline \multirow[t]{2}{*}{ Interacción } & Posee un sistema de ayuda & $\begin{array}{l}\text { (GYANT, 2021; La Previsora S.A., 2020; Línea de } \\
\text { emergencias de Bogotá, 2007; Medical ID, 2021; S.O.S } \\
\text { Emergencias, 2020; Safe365, 2021; Seguros Bolívar, 2020; } \\
\text { Sura, 2017). }\end{array}$ \\
\hline & $\begin{array}{l}\text { Cuenta con localización en } \\
\text { tiempo real }\end{array}$ & $\begin{array}{l}\text { (Comunidad de Madrid, 2015; International SOS, 2021; Línea } \\
\text { de emergencias de Bogotá, 2007; Ministerio del Interior, 2016; } \\
\text { S.O.S Emergencias, 2020; Safe365, 2021; Seguros Bolívar, } \\
\text { 2020; Sura, 2017). }\end{array}$ \\
\hline \multirow[t]{2}{*}{ Público objetivo } & General & $\begin{array}{l}\text { (App Primeros Auxilios, 2014; Comunidad de Madrid, 2015; } \\
\text { GYANT, 2021; International SOS, 2021; La Previsora S.A., } \\
\text { 2020; Línea de emergencias de Bogotá, 2007; Medical ID, } \\
\text { 2021; Ministerio del Interior, 2016; S.O.S Emergencias, 2020; } \\
\text { Safe365, 2021; Seguros Bolívar, 2020; Sura, 2017). }\end{array}$ \\
\hline & Con conocimiento previo & (GYANT, 2021; MDCalc, 2021). \\
\hline \multirow[t]{5}{*}{$\begin{array}{l}\text { Servicios } \\
\text { integrados }\end{array}$} & Servicio médico & $\begin{array}{l}\text { (Comunidad de Madrid, 2015; GYANT, 2021; International } \\
\text { SOS, 2021; Línea de emergencias de Bogotá, 2007; Ministerio } \\
\text { del Interior, 2016; S.O.S Emergencias, 2020; Safe365, 2021; } \\
\text { Sura, 2017). }\end{array}$ \\
\hline & Servicio de policía & $\begin{array}{l}\text { (Comunidad de Madrid, 2015; International SOS, 2021; Línea } \\
\text { de emergencias de Bogotá, 2007; Ministerio del Interior, 2016; } \\
\text { S.O.S Emergencias, 2020; Safe365, 2021). }\end{array}$ \\
\hline & Servicio de bomberos & $\begin{array}{l}\text { (Comunidad de Madrid, 2015; International SOS, 2021; Línea } \\
\text { de emergencias de Bogotá, 2007; Ministerio del Interior, 2016; } \\
\text { S.O.S Emergencias, 2020; Safe365, 2021). }\end{array}$ \\
\hline & Seguro para vivienda & (La Previsora S.A., 2020; Sura, 2017). \\
\hline & Seguro para vehículo & (La Previsora S.A., 2020; Seguros Bolívar, 2020; Sura, 2017). \\
\hline
\end{tabular}

De la Tabla 1 se puede concluir que, si bien existen aplicaciones que cubren los servicios de emergencias médicas, en cuanto a personalización son pocos los trabajos relacionados que almacenan información básica de los usuarios y sólo uno el que almacena la información médica (Medical ID, 2021). La única información 
que es usada para generar valor a los usuarios es la de sus contactos de emergencia y las pólizas de seguros. Pasando al apartado de servicios, sólo una aplicación brinda sugerencias de entidades que puedan atender la emergencia del usuario; sin embargo, no es gratuita y, además, muestra únicamente las entidades que tienen un convenio con dicho sistema. De igual manera, tampoco hay un filtro especializado que asegure al usuario que las sugerencias son acordes a su necesidad.

Por todo lo anterior, surge la necesidad de crear EmergenciApp, una aplicación que permite orientar al usuario hacia qué IPS puede dirigirse ante una emergencia y notificar a sus familiares o amigos sobre su ubicación. Esta aplicación cuenta con fortalezas tales como:

- Utiliza datos del usuario y del contexto con el fin de proveer servicios ajustados a su realidad, tales como: redireccionar al usuario a la IPS más apropiada de acuerdo con su emergencia y avisar a sus seres queridos sobre el lugar a donde fue remitido.

- Maneja diversos tipos de emergencia: médicas, de seguridad, domésticas, entre otras.

- Ofrece una interfaz usable que permite mostrar los servicios enriquecidos, que puede ser usada fácilmente por diversos tipos de usuarios.

- Determina, basada en la información del usuario, particularmente en su historia clínica y en su tipo de emergencia, la IPS más adecuada para su tratamiento. Todo esto a partir de una serie de reglas consultadas y avaladas por profesionales de la salud.

A continuación, se describe brevemente EmergenciApp, así como la manera en que fue diseñado y desarrollado.

\section{EmergenciApp}

EmergenciApp es una aplicación móvil que responde a diferentes tipos de emergencias que puede presentar toda persona residente en Bogotá. Esta aplicación está al servicio de la persona que requiera de atención de emergencias médicas y que no sepa a qué IPS acudir. El principal objetivo del sistema propuesto es brindar una serie de servicios personalizados, entendiendo por esto, aquellos que se adaptan a las necesidades y el contexto en el cual se encuentre el usuario. Los servicios que ofrece la aplicación son los listados a continuación:

a. Sugerir al usuario IPS.

b. Notificar a miembros del grupo familiar.

c. Permitir llamadas a los números de emergencia.

Para esto, EmergenciApp cuenta con un modelo de personalización que enriquece sus servicios. Éste se puede apreciar en la Figura 1. El árbol de perfil de usuario muestra los datos del usuario que permiten brindar una mejor experiencia en cuanto a servicios personalizados. Es por ello, que se tienen en cuenta los datos básicos y las preferencias del usuario dentro de la aplicación. Principalmente, sus datos médicos además de las pólizas de seguro y el grupo familiar del mismo; igualmente, se consideran los datos del usuario sobre sus principales preferencias frente a las IPS de la ciudad lo que contribuye a que el sistema lo re-direccione a la(s) que mejor se adapte(n) a sus necesidades. Es por ello que los datos recolectados por el sistema, desde su ubicación y condición de salud hasta los datos que componen la información básica de su emergencia, son la base para encontrar soluciones adecuadas a la necesidad del usuario. 


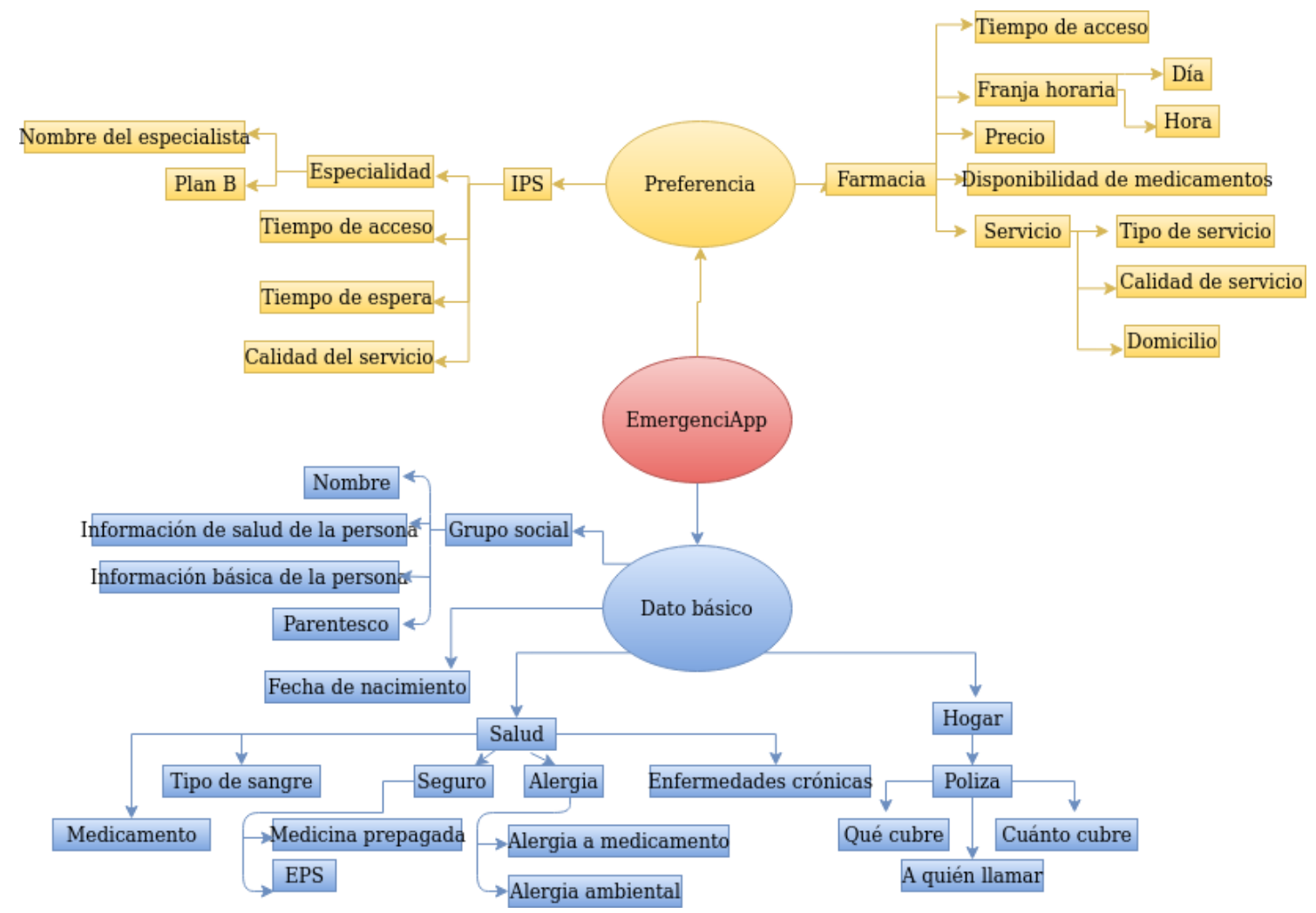

Figura 1. Árbol de perfil de usuario

Debido a que el principal atractivo de la propuesta recae en el proceso de re-direccionamiento, se dedicará esta sección a detallar este sistema que busca sugerir al usuario la IPS más adecuada para tratar su emergencia de salud, además, del posterior re-direccionamiento hacia dicha entidad. En primer lugar, EmergenciApp determina los especialistas necesarios para el caso del usuario debido a que el objetivo es que éste no sea redireccionado a otra IPS más allá de la recomendada. Si bien la emergencia puede no requerir de un proceso de interconsulta (donde el médico urgenciólogo determina que es necesario el apoyo de un especialista para dicho caso particular), es imposible determinar la gravedad de lo ocurrido, pues esto es un trabajo que se asemeja a un Triage, un proceso muy delicado de hacer desde un dispositivo móvil al no contar con cierto tipo de sensores para medir signos vitales del usuario, además de que no es el enfoque que se busca dar. Cabe mencionar que este proceso fue asesorado por profesionales médicos.

El primer paso para determinar qué especialidad necesitará el usuario en caso de requerir un proceso de interconsulta en la IPS que se le recomiende, será determinar el tipo de afección que tiene, si es por una quemadura, ya sea por exposición de la piel a químicos, electricidad y fuego; intoxicaciones, ya sea por drogas o alimentos en mal estado; lesiones, ya sea por cortadas, fracturas, etc., y síntomas varios, donde se incluye todos aquellos padecimientos que no entran en las otras categorías, como lo puede ser la visión borrosa, temblores, dolores sin origen aparente y demás.

Una vez se conoce el tipo de afección, se busca determinar el área afectada, lo cual se dividió en cuatro partes: cabeza y cuello; tórax y abdomen superior; abdomen inferior y pelvis y, finalmente, extremidades. Claramente estas áreas aún abarcan muchos posibles sistemas u órganos y, por ende, varios especialistas involucrados, por lo que se debe acotar aún más la zona afectada.

También, en caso de determinar que el usuario tiene comprometida la cabeza y el cuello, por ejemplo, se busca determinar con más exactitud qué partes el usuario identifica como afectadas, por lo que, para este caso particular de la cabeza, se necesita determinar cuál es la sub-área afectada entre las cuales se encuentra el cerebro, los ojos, la nariz, los oídos, la boca y la garganta.

Finalmente, lo último que queda es determinar los síntomas principales y asociados, por lo que, para cada área afectada, se puede sintetizar un conjunto de síntomas que el usuario puede experimentar. Por ejemplo, en el caso de la garganta, se puede experimentar tos, tos con sangre, dolor, entre otros. Determinar esto es crucial, pues dependiendo de los síntomas, es posible que el especialista requerido sea diferente. 
Ahora, para determinar todo esto se definió un sistema de preguntas que el usuario responderá al momento de reportar una emergencia, por lo que se le mostrará un conjunto de preguntas secuenciales las cuales se organizan a modo de árbol como se puede observar en Figura 2, donde éstas están distribuidas en cinco niveles: ¿Qué sucedió? con el fin de determinar el tipo de afección, ¿Cuál es el área afectada?, ¿Cuál es la sub-área afectada?, ¿Cuál es el síntoma principal? y, ¿Cuál es el síntoma asociado? El camino denotado en amarillo es un ejemplo de las preguntas que podría responder el usuario.

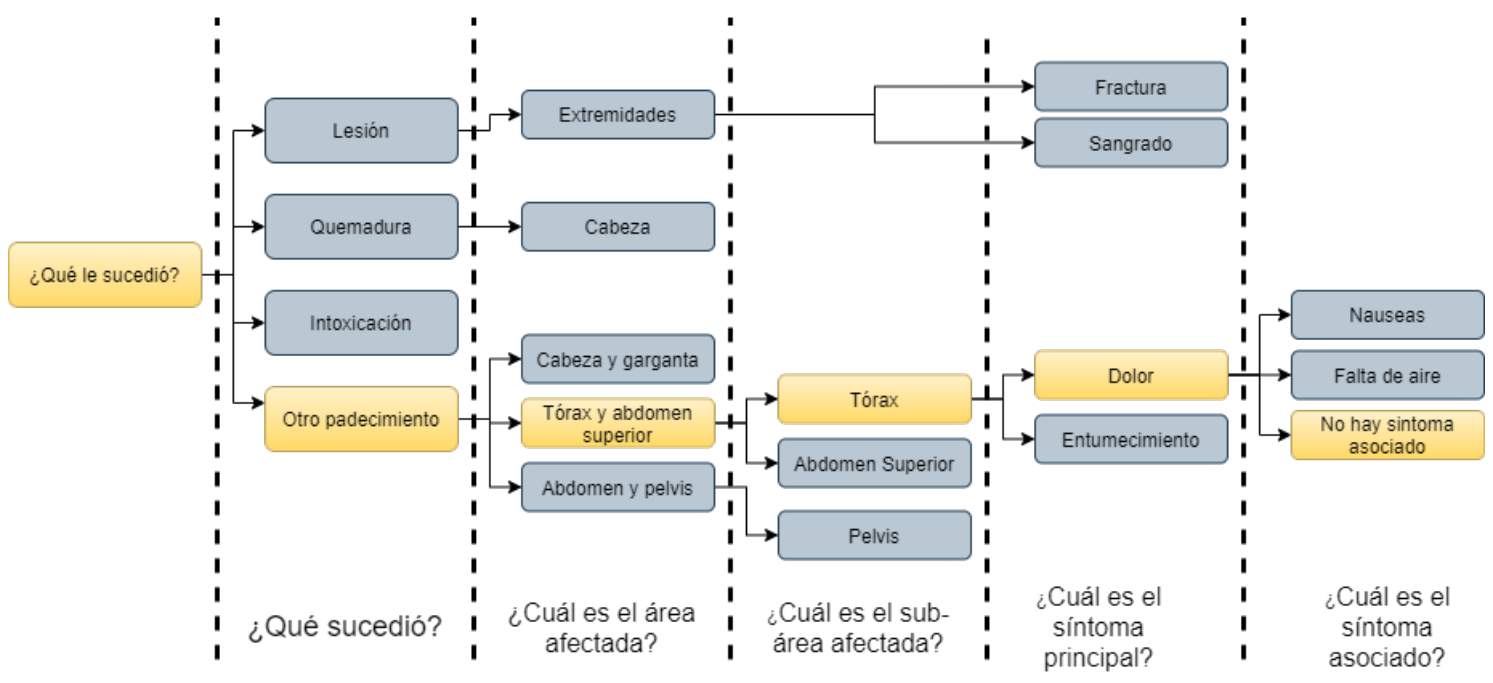

Figura 2. Árbol de preguntas

Una vez se ha respondido estas preguntas, el sistema define qué especialidades son requeridas para los síntomas del usuario, por ejemplo, si tiene un dolor en la garganta, se le debe recomendar una IPS que tenga otorrinolaringología. Sin embargo, un aspecto crucial a tener en cuenta es que, si bien hay padecimientos que se manifiestan en cierta parte del cuerpo, su origen puede ser en otra. Ejemplo de esto es una trombosis cerebral, en cuyos síntomas está la visión borrosa. Si el usuario en la aplicación selecciona que como síntoma tiene visión borrosa, EmergenciApp deberá tener en cuenta que esto es algo que se puede producir a razón de la trombosis cerebral, y, por ende, recomendar una IPS que tenga tanto oftalmología como neurología para este caso particular.

El siguiente paso, luego de determinar las especialidades necesarias, consta de listar las IPS candidatas, aplicando un conjunto de filtros que se definen en la Figura 3, donde la IPS que se recomienda al final será la que, en primer lugar, cumpla con los requerimientos de convenios con los seguros médicos del usuario. $\mathrm{Si}$, por ejemplo, se tiene un seguro con la EPS, EmergenciApp filtrará y mostrará únicamente las IPS que tengan convenio con esta EPS. Las que no cumplan con este requisito, automáticamente quedan descartadas a razón de que re-direccionar al usuario a algunas de éstas puede llevar a que éste no sea atendido para procesos de interconsulta o exámenes médicos que puedan solicitar. Lo siguiente, es declarar las IPS que no atiendan al usuario según su edad. En este sentido, lo que se busca es evitar que a un adulto se le sugiera un hospital que sólo atienda a niños. Finalmente, el filtro de especialidades se formula con lo expuesto anteriormente mediante la determinación de los médicos especialistas que requerirá el usuario. Luego de esto, las IPS restantes se ordenarán según tres criterios por prioridad: la preferencia del usuario por la IPS, el tiempo de acceso desde la ubicación del usuario a la misma, y la opinión de otros usuarios sobre la IPS. Finalmente, se mostrarán las IPS más adecuadas para atender la emergencia particular del usuario. 


\section{Filtrado y ordenamiento de IPS candidatas}

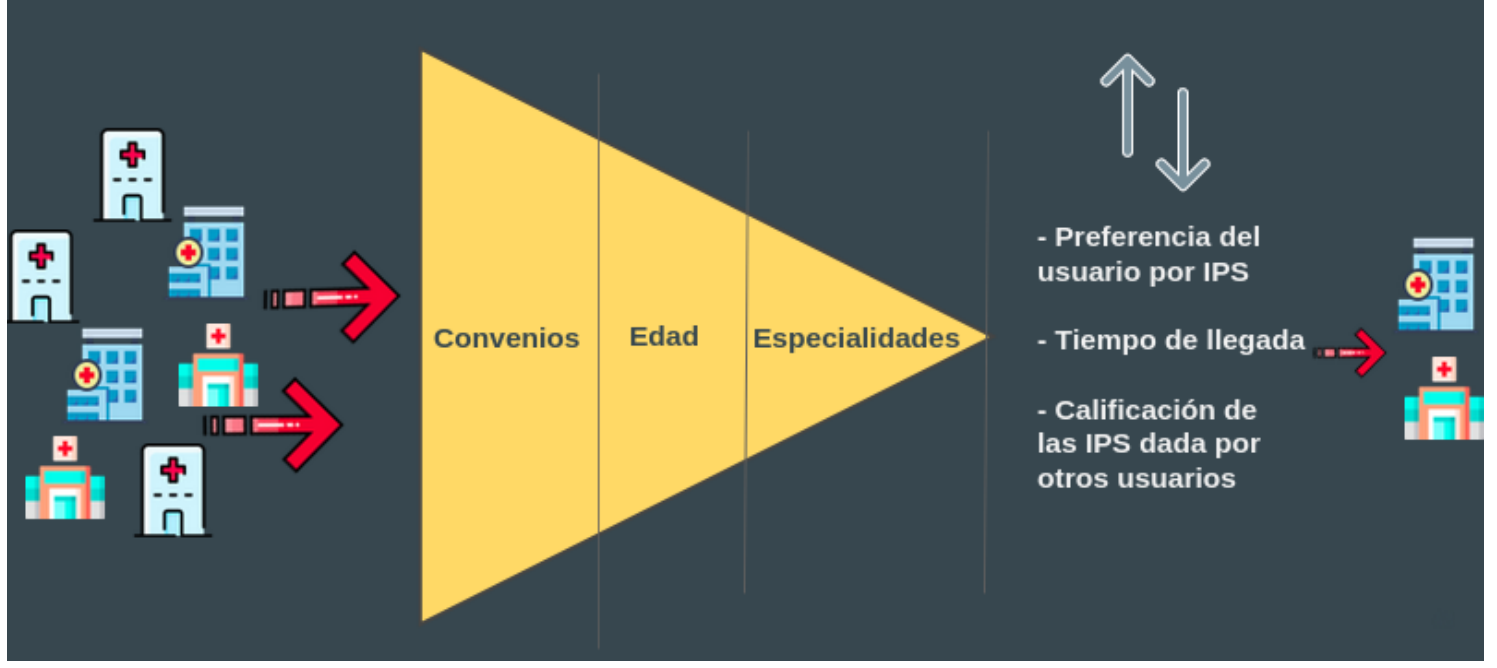

Figura 3. Sistema de filtrado de IPS

En el siguiente diagrama BPMN (ver Figura 4), se puede apreciar el proceso que realiza el sistema para mostrarle las IPS más adecuadas al usuario una vez son aplicados el conjunto de filtros y la priorización de información con los criterios definidos previamente en esta sección para las emergencias de salud.

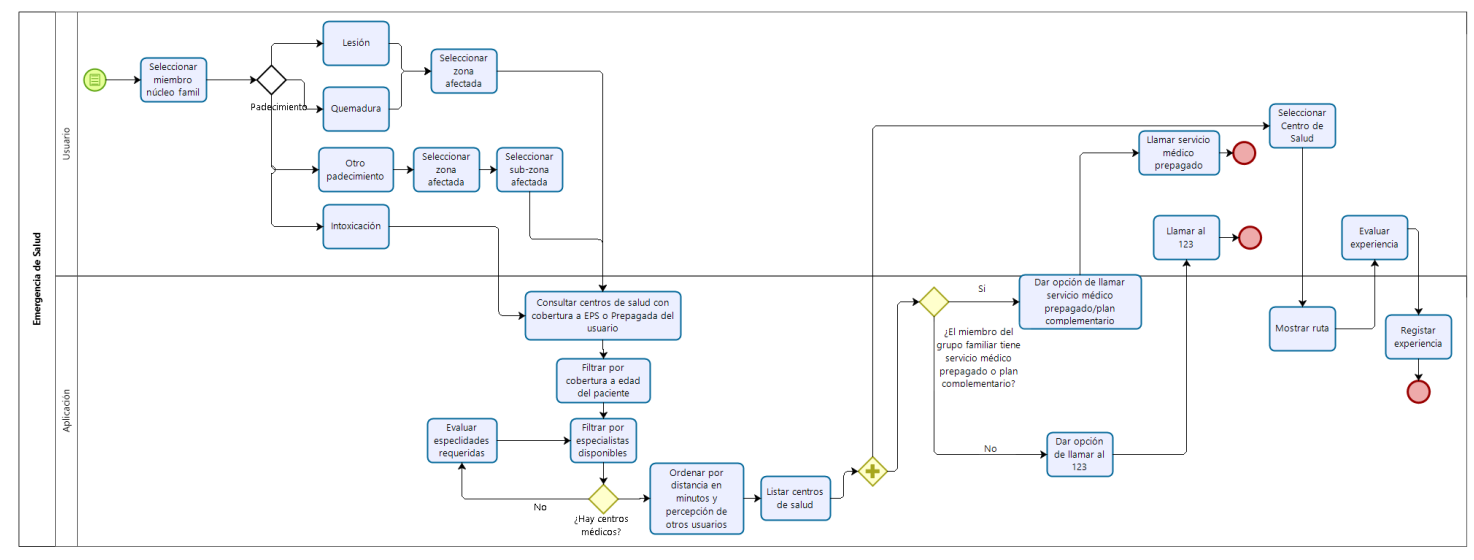

Figura 4. Diagrama BPMN - Emergencias de Salud

\section{Diseño y desarrollo de EmergenciApp}

En esta sección se describe brevemente el diseño y desarrollo de EmergenciApp. Sólo se mostrarán los diagramas más relevantes por cuestiones de espacio.

El diagrama de casos de uso es presentado en la Figura 5. los casos de uso en amarillo son los relacionados con la personalización. Dentro de los casos de uso sobresale que el usuario puede reportar emergencias de salud, policiales, bomberos, y caseras, obteniendo la información respectiva sobre los centros de atención de emergencias distritales y privados que pueden atender la emergencia del usuario acorde con sus datos personales y de la emergencia en sí. Además, los usuarios pueden crear un grupo 
familiar, reportar emergencias para ellos, y notificarlos en caso de que él/ella tenga una emergencia. La personalización de los casos de uso está basada en que estos se pueden realizar de acuerdo con la información que el usuario ingresa en el momento de registrarse en la aplicación, y con los datos que reporte de la emergencia. Por esta razón, estos casos de uso brindan información al usuario sobre los centros de atención de emergencias, una vez se ha procesado su información básica.

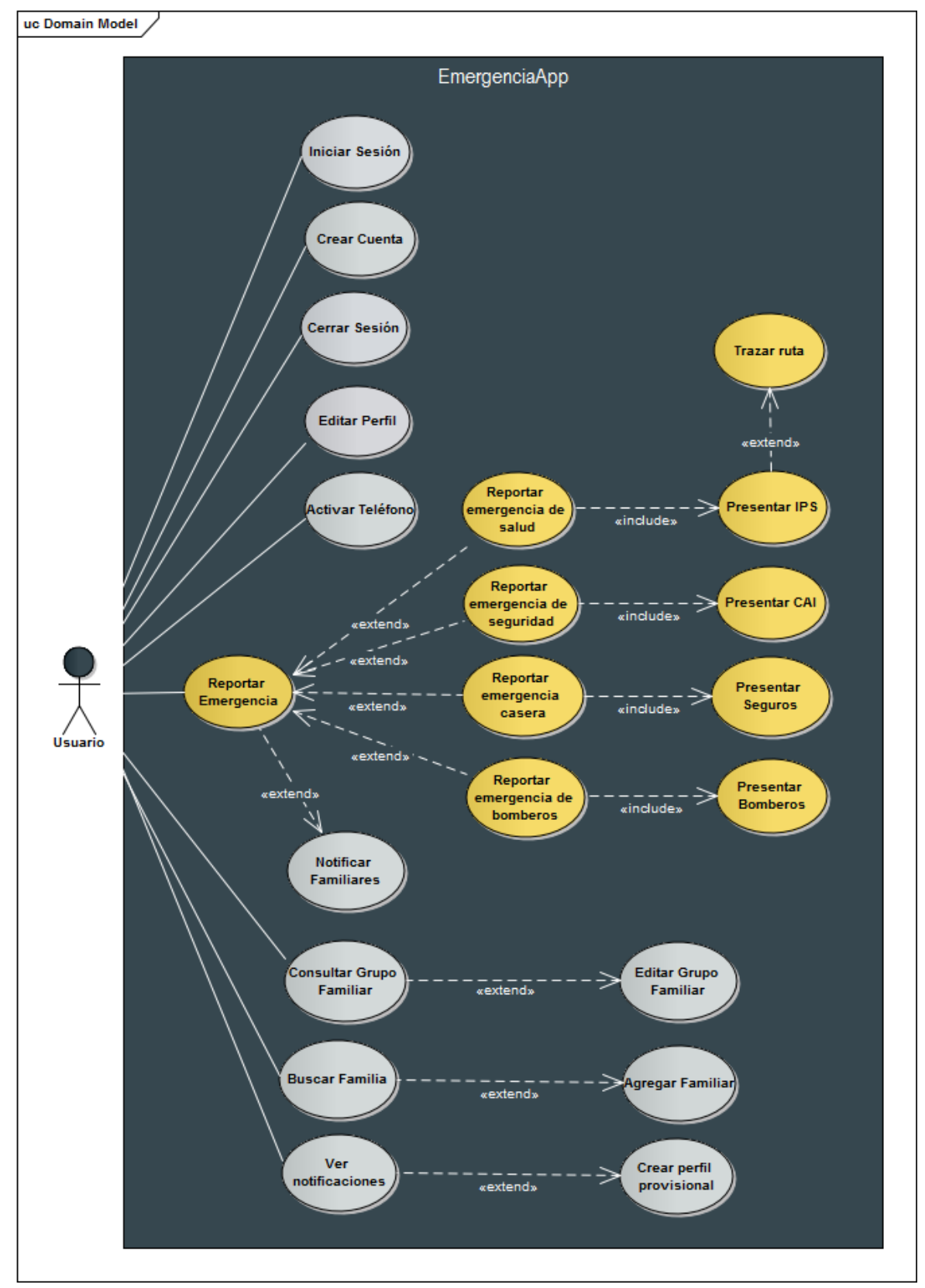

Figura 5. Diagrama de casos de uso

En la Figura 6 se evidencia el diagrama de clases de EmergenciApp, el cual contiene todos los atributos y entidades relevantes para la implementación técnica del proyecto. Allí se encuentran adicionalmente las relaciones de los objetos que hacen posible el funcionamiento esperado del sistema de re-direccionamiento de emergencias. 


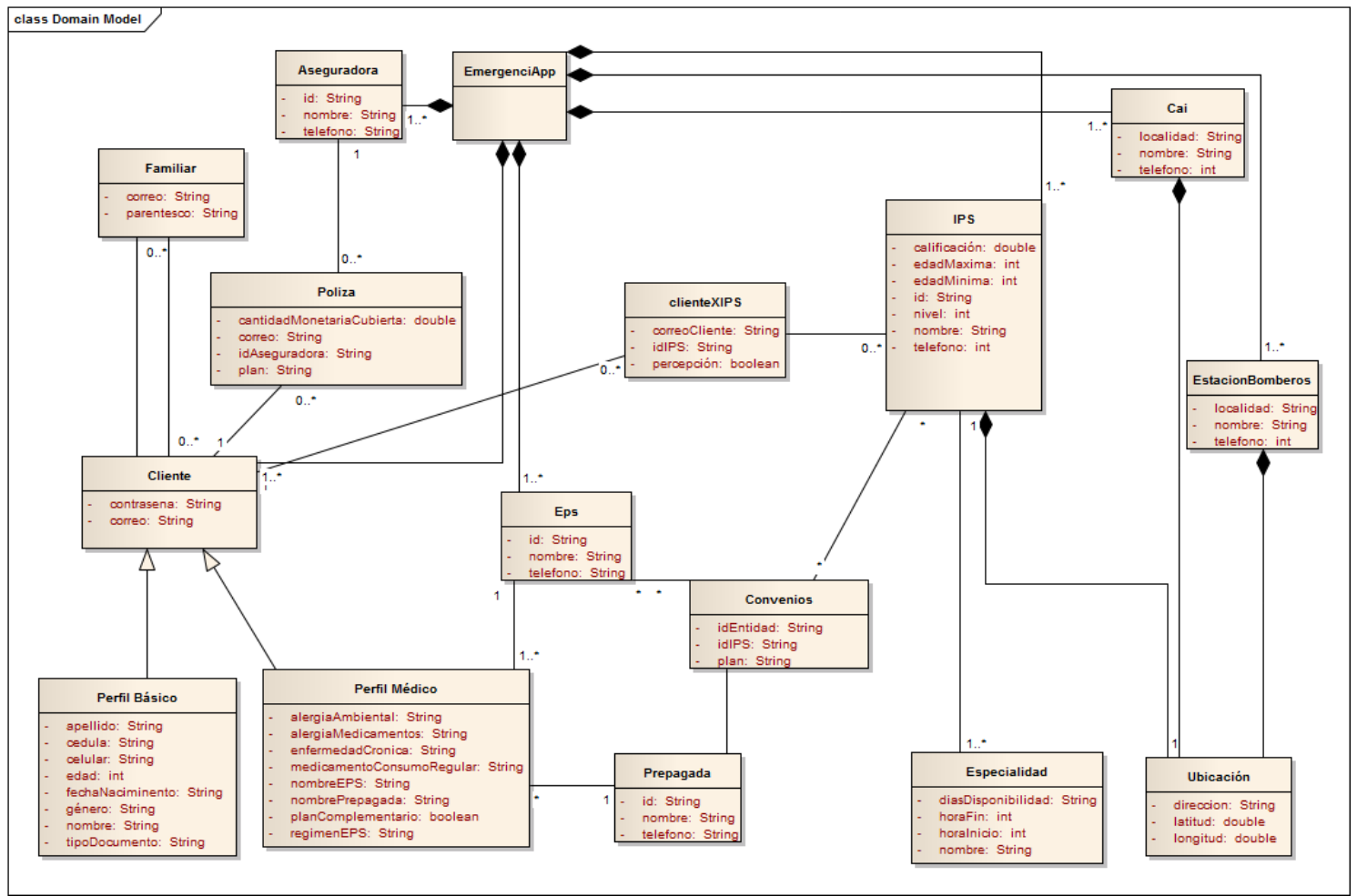

Figura 6. Diagrama de clases

Luego, se propuso dos vistas arquitectónicas que favorecen los siguientes atributos de calidad ordenados según su prioridad: disponibilidad, usabilidad, desempeño, y seguridad. Por esto, el primer diagrama, el de componentes que se puede observar en Figura 7 se separó en tres: EmergenciApp App, EmergenciApp WebServer, y Database EmergenciApp. Después de esto, se planteó el diagrama de despliegue que muestra cómo quedaría EmergenciApp puesto en nodos físicos. Como se puede observar en la Figura 8, el componente de presentación desarrollado en Android Studio quedaría en el dispositivo del usuario, mientras que la lógica de negocio, desarrollada en Java EE, se alojaría en un servidor dedicado, y la base de datos, que al trabajarse con Firebase (Firebase, 2020), estaría alojado en la nube.
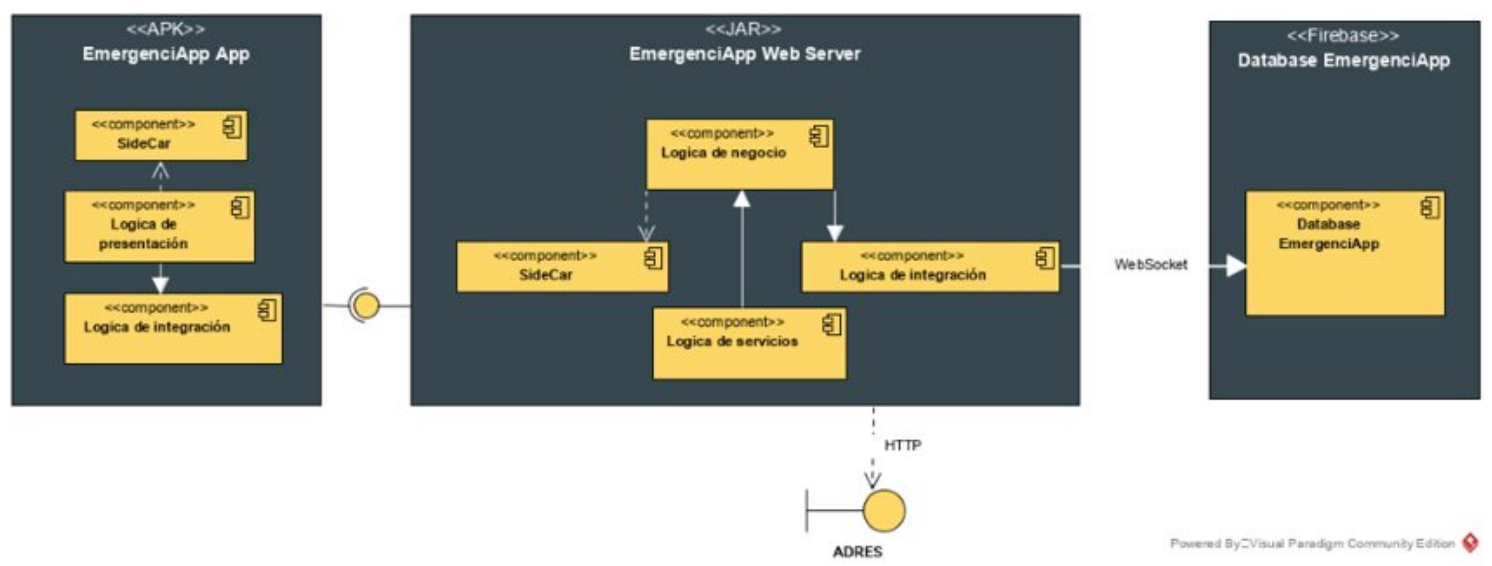

Figura 7. Diagrama de componentes 


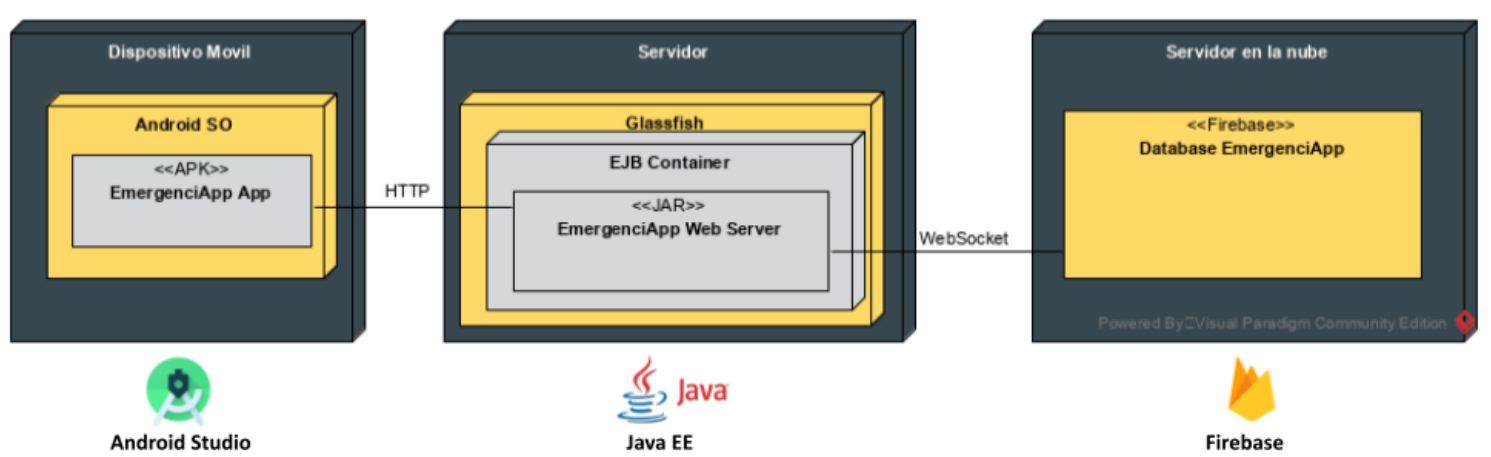

Figura 8. Diagrama de despliegue

Para mostrar la implementación de EmergenciApp, se presenta un conjunto de pantallas que detallan sus funcionalidades. La pantalla principal, muestra lo que observará todo usuario una vez registrado en el sistema (ver Figura 9), donde el botón señalado con el número 1, indica la opción de reportar una emergencia, el número 2 es para solicitar ayuda del 123 mediante una llamada telefónica, el número 3 es para consultar y editar el perfil, el número 4 es para consultar y modificar su grupo familiar, y finalmente el número 5, es para visualizar las notificaciones al usuario.

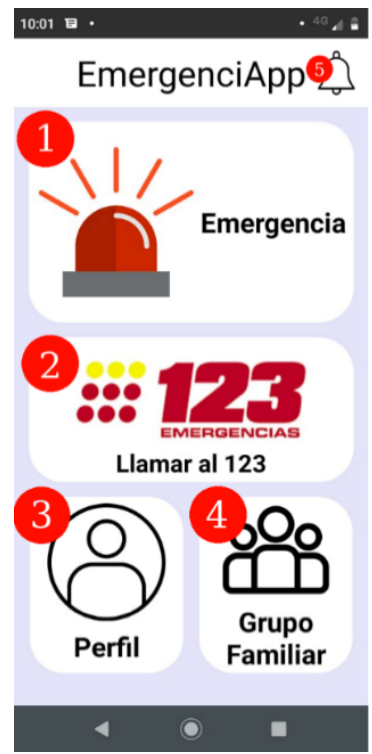

Figura 9. Pantalla principal

Ya en el momento en el que el usuario reporte una emergencia, se le muestran las pantallas presentadas en la Figura 10, donde empieza a funcionar el árbol de preguntas de la sección 4, cuyo fin es el de determinar las especialidades requeridas por el usuario. 

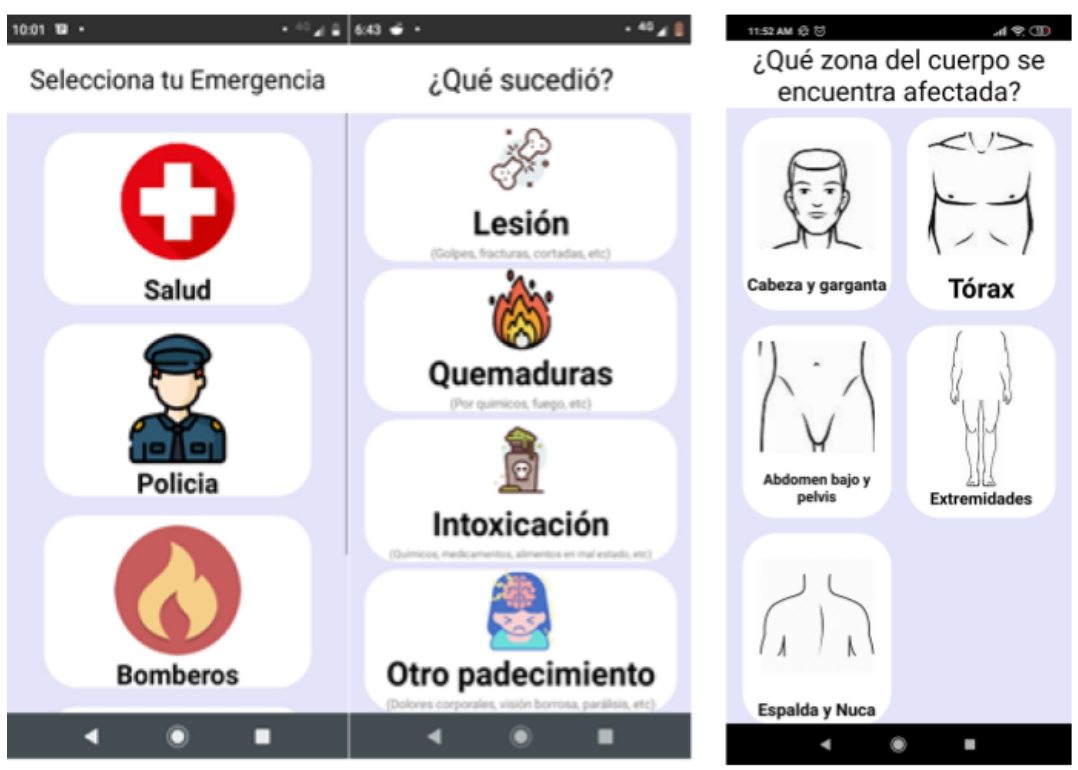
¿Qué parte de la cabeza se encuentra afectada?

Figura 10. Pantallas descripción emergencia

Por último, en la Figura 11 se puede observar la lista de las IPS filtradas para que el usuario seleccione la que más le convenga, donde al seleccionarla, se le mostrará la ruta más adecuada para llegar a ésta.

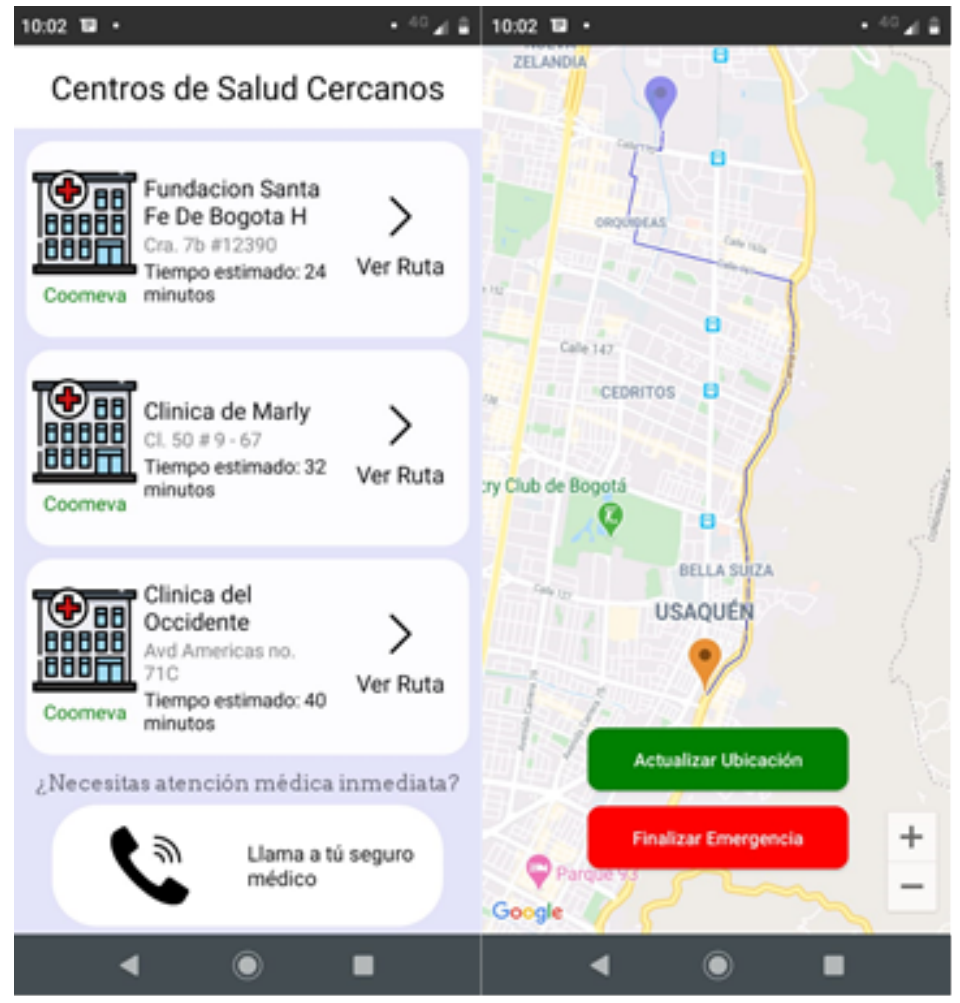

Figura 11. Pantallas re-direccionamiento emergencia 


\section{Pruebas}

Se realizaron encuestas a diferentes grupos poblacionales de edades con el fin de conocer sus principales comportamientos en situaciones de emergencias. Esta encuesta fue aplicada a 93 personas y las principales conclusiones fueron:

- La prioridad de los encuestados (57\%) es la atención en centros hospitalarios que tengan convenios con su EPS, además de una buena atención $(41,9 \%)$, y su cercanía con el centro hospitalario $(39,8 \%)$.

- Un $81,7 \%$ de los encuestados estaría dispuesto a utilizar una aplicación que le brinde información sobre las entidades que pueden atenderlo ante una emergencia.

Adicionalmente, se realizaron pruebas de usabilidad sobre el prototipo funcional para emergencias de salud de EmergenciApp. Estas pruebas se aplicaron a un grupo de siete personas, debido a que, en el momento de realizarlas, se presentaba a nivel global la pandemia COVID-19, lo cual dificultó trabajar sobre un mayor número de personas. Para la obtención de resultados de las pruebas de usabilidad se realizaron 10 preguntas basadas en el estándar Questionnaire for User Interface Satisfaction QUIS (Perlman, 1988) y las heurísticas para evaluar usabilidad formuladas por Jakob Nielsen (Nielsen, 1994). A partir de los resultados obtenidos se pudo observar que los usuarios se encontraron muy satisfechos con la utilidad de la aplicación, la facilidad de aprender el sistema, y la eficacia con la que se pueden realizar las tareas de la aplicación. Como aspectos de mejora se tuvo en cuenta que el sistema podría ser más atractivo visualmente, además de mejorar el manejo y prevención de errores.

Adicionalmente, los expertos en usabilidad, quienes también realizaron estas pruebas, encontraron que las principales fortalezas del prototipo son la facilidad de aprender, lo atractiva e intuitiva que es su interfaz gráfica, y la eficacia con la que se pueden realizar las funcionalidades del sistema. Como aspectos a mejorar los expertos resaltaron el manejo y la prevención de errores dentro del sistema, y la satisfacción general de los usuarios con la aplicación. Los resultados de las pruebas de usabilidad se pueden apreciar en Figura 12.

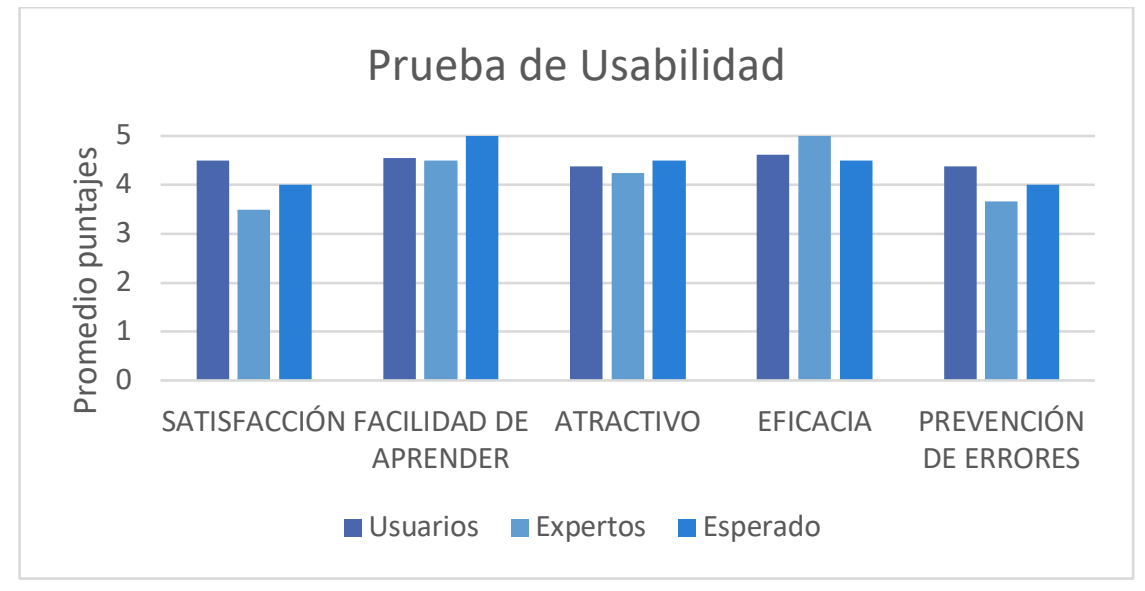

Figura 12. Resultados pruebas usabilidad

También, se realizaron pruebas de personalización al prototipo de la aplicación con un mismo perfil de usuario. Con ello se buscaba definir los resultados que arrojaba el sistema cuando se tiene en cuenta la información de los seguros médicos del usuario, las especialidades requeridas para atender la emergencia presentada, y cuando ninguna información es tenida en cuenta para realizar este proceso.

En Figura 13(a) se puede apreciar los resultados sin personalización de una emergencia de salud del usuario. En ella se puede visualizar que el sistema muestra todas las IPS que se tienen registradas en el sistema sin ningún tipo de prioridad, por ejemplo, los centros médicos mostrados en dicha figura no están organizados por el tiempo estimado de llegada hasta el centro hospitalario. En cambio, en Figura 13(b) se puede observar que los centros médicos están debidamente organizados acorde con los criterios definidos en la sección 4, según la afiliación de los seguros médicos del usuario, las especialidades requeridas para 
atender la emergencia, y la distancia a la que se encuentra de ellos, además de la preferencia del usuario por los centros médicos, entre otros.

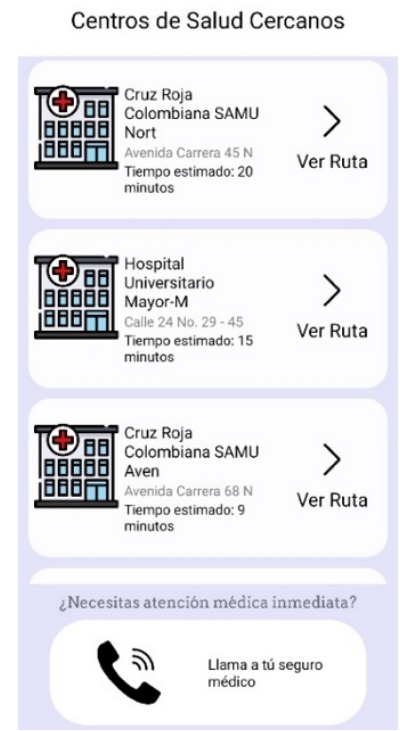

(a)

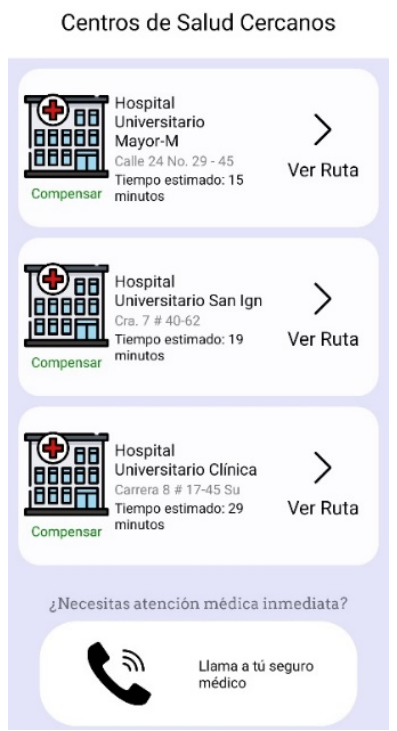

(b)

Figura 13(a). Resultados sin personalización, Figura 13(b). Resultados con personalización

Otra prueba que se realizó fue para usuarios del mismo núcleo familiar, pero con edades y necesidades diferentes (ver Figura 14):

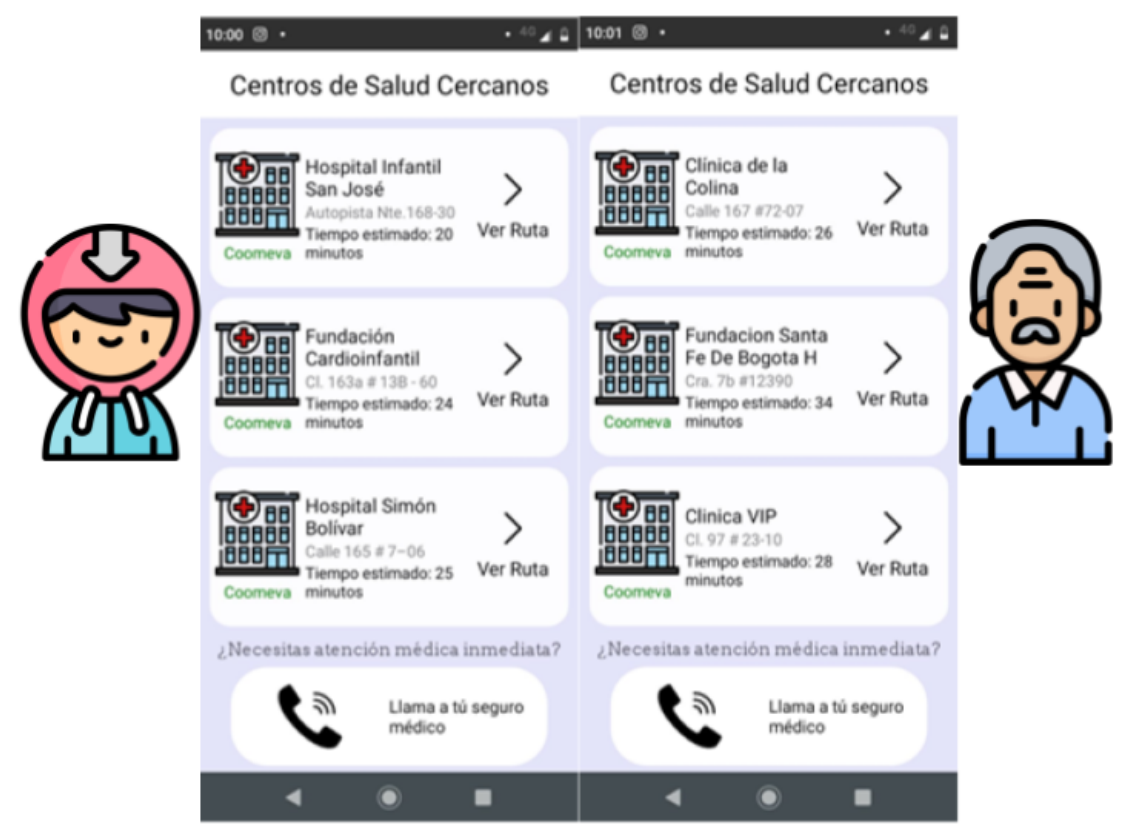

Figura 14. Pruebas con usuarios del mismo grupo familiar, con edades y necesidades diferentes

También, con usuarios con el mismo re-direccionamiento (IPS) aunque ubicados en diferentes localidades (ver Figura 15): 


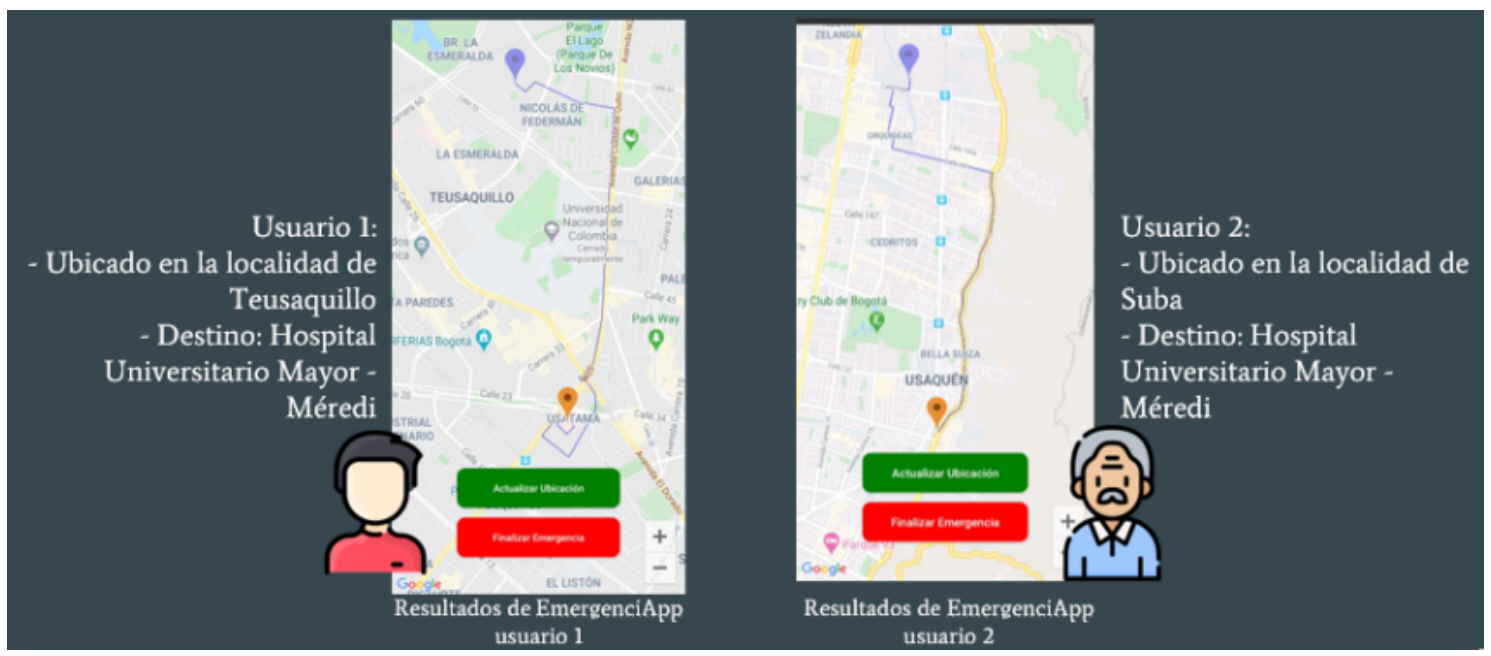

Figura 15. Pruebas con usuarios hacia la misma IPS, con diferente localización

Finalmente, cuando un usuario ingresa por primera vez a EmergenciApp, y no tiene ninguna preferencia, se aplican los filtros como se indicó en la sección 4. Sin embargo, ante una quemadura en la cabeza y ya habiendo seleccionado como preferida una IPS, el sistema la señala como primera opción (sólo si atiende este tipo de emergencia). Después, a pesar de tener una preferencia, pero si esa IPS no atiende, por ejemplo, una intoxicación, lo remite a la IPS que puede atender mejor su emergencia (ver Figura 16).

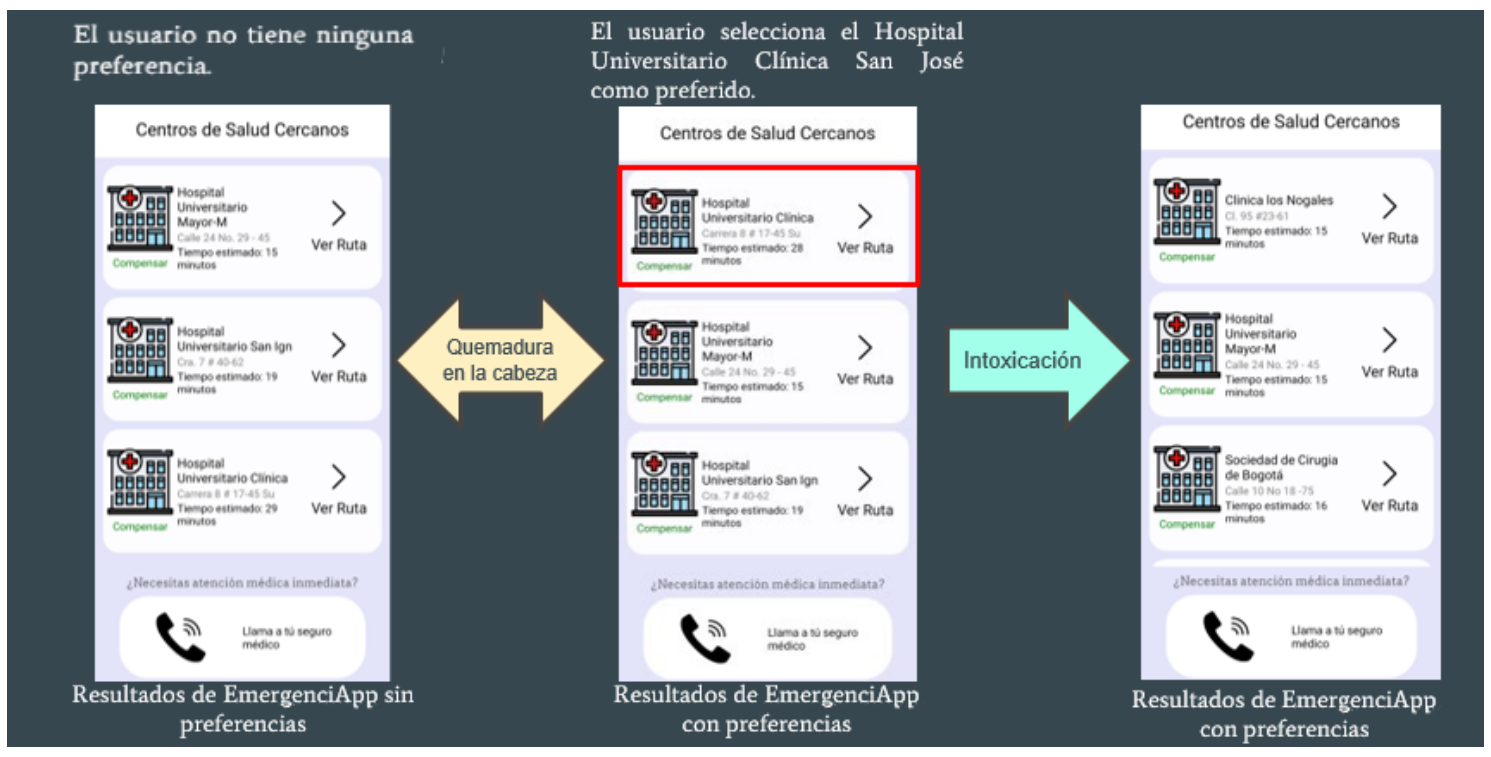

Figura 16. Mismo usuario, diferentes re-direccionamientos debido al tipo de emergencia

\section{Conclusiones y trabajo futuro}

En este artículo se presentó EmergenciApp, una aplicación móvil que re-direcciona un usuario hacia la IPS que pueda atender de mejor manera una emergencia médica, basada en sus necesidades y características. El proceso de re-direccionamiento en casos de emergencias y acceso a información de las entidades que pueden ayudar en este tipo de sucesos, da una aproximación real sobre cómo se podría implementar un proceso eficiente y de gran ayuda a los residentes de la ciudad de Bogotá y de cualquier otra ciudad (ver Figura 17). Además, se estableció un modelo de personalización que permite adaptar los servicios según las necesidades y datos básicos de cada usuario. Todo esto fue logrado mediante el trabajo en conjunto con profesionales de la salud lo que contribuyó a determinar los padecimientos del usuario. Asimismo, se resalta 
la importancia de contar con el apoyo de expertos en la problemática a solucionar, pues es con base en sus sugerencias y experiencia que se logra que este tipo de proyectos, puedan aportar algo significativo y útil.

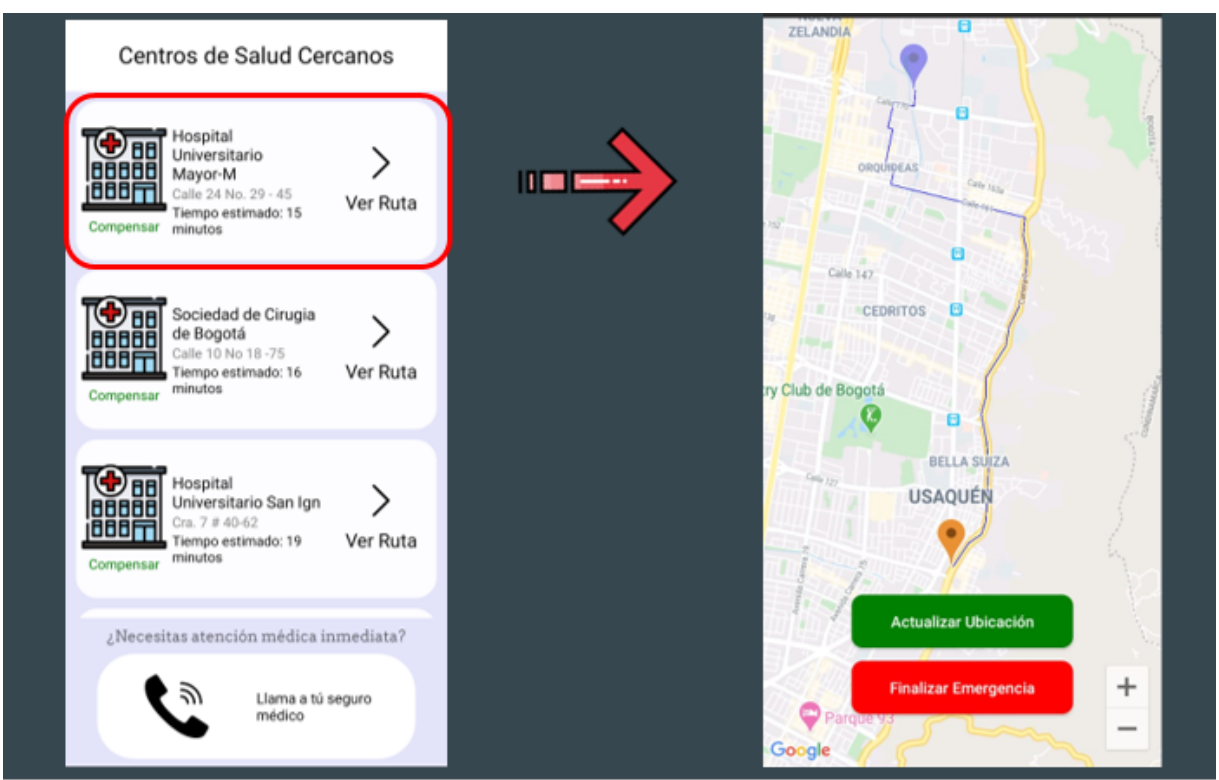

Figura 17. Re-direccionamiento a IPS basado en características y preferencias del usuario, así como generación de ruta desde su ubicación actual a la IPS

Finalmente, se creó un sistema personalizable que ofrece servicios diferentes según cada usuario. Cabe mencionar que, debido a que se planteó como desarrollo móvil, se habilita a este sistema para que funcione en entornos nómadas, donde la información se puede acceder donde sea y a la hora que sea. En resumen, las fortalezas de EmergenciApp son:

- Utiliza datos del usuario y del contexto con el fin de proveer servicios ajustados a su realidad, todo esto basado en el perfil de usuario y la definición misma de la emergencia.

- Maneja diversos tipos de emergencia: médicas, de seguridad, domésticas, entre otras. Cabe mencionar que este artículo se enfocó sólo en las de salud.

- Ofrece una interfaz usable que permite mostrar los servicios enriquecidos y utilizar fácilmente la aplicación a diversos tipos de usuarios. Esto se manifestó en las pruebas con usuarios finales, en las que, a usuarios diferentes, les mostraba diferentes resultados.

- Determina, basada en la información del usuario, particularmente en su historia clínica y en su tipo de emergencia, la IPS más adecuada para su tratamiento, todo esto determinado por una serie de reglas consultadas y avaladas por profesionales de la salud. Esto demuestra la rigurosidad seguida y el trabajo interdisciplinar que tiene el módulo de emergencias médicas.

Como trabajo futuro, se contempla enriquecer el proceso de pre-diagnóstico mediante el asesoramiento continuo de profesionales de la salud con el fin de tener una mayor precisión en la determinación de especialidades requeridas por los usuarios. Para ello, se manejará un motor de inferencia y la participación de profesionales de diversas especialidades para refinar este pre-diagnóstico. Esto se logrará completando el árbol de preguntas de la sección 4, más específicamente, aquellas relacionadas con los síntomas principales y asociados ya que es un trabajo muy extenso al considerar la cantidad de síntomas y padecimientos que puede haber. Finalmente, gracias a la arquitectura modular, se podrá ingresar los componentes relacionados con otros tipos de emergencias y se establecerán acuerdos con las instituciones para alimentar el sistema con información actualizada sobre los convenios entre EPS e IPS, aseguradoras y la línea 123 (junto con las que se manejen para atención de emergencias en cada ciudad). 


\section{Declaración de conflicto de intereses}

Los autores declaran no tener conflicto de intereses con respecto a la investigación, autoría o publicación de este artículo.

\section{Financiación}

Los autores no recibieron apoyo financiero para la investigación, autoría y/o publicación de este artículo.

\section{ORCID iD}

\section{Ángela Carrillo-Ramos iD https://orcid.org/0000-0001-9086-5945}

\section{Referencias}

App Primeros Auxilios. (2014). Primeros auxilios fáciles. http://www.primerosauxiliosfaciles.es Caracol Radio. (2018). El 'Paseo de la muerte' ya deja 89 víctimas durante 2018. https://caracol.com.co/radio/2018/10/03/nacional/1538571677_077170.html

Comunidad de Madrid. (2015). App de Emergencias. http://www.madrid.org/112/index.php/actualidad/app-deemergencia

El Espectador. (2018). Siete de cada 10 llamadas a la Línea 123 son falsas. https:/www.elespectador.com/noticias/bogota/siete-de-cada-10-1lamadas-a-la-linea-123-son-falsas/

Firebase. (2020). Firebase helps you build and run successful apps. https://firebase.google.com

GYANT. (2021). GYANT makes people better. https://gyant.com/

International SOS. (2021). Assistance App. https://www.internationalsos.com/assistance-app

La Previsora S.A. (2020). La Previsora Seguros. https://apps.apple.com/ca/app/la-previsora-seguros/id1515617326

Línea de emergencias de Bogotá. (2007). ¿Qué es la Línea 123? https://scj.gov.co/landing/linea-123/

MDCalc. (2021). MD+CALC. https://www mdcalc.com

Medical ID. (2021). Main features. https://medicalid.app

Ministerio del Interior. (2016). Emergencia 9-1-1 Aplicación para teléfonos celulares. https://www.minterior.gub.uy/index.php/component/content/article?id=4385

Nielsen, J. (1994). 10 Usability Heuristics for User Interface Design. https:/www.nngroup.com/articles/ten-usabilityheuristics/

Perlman, G. (1988). Questionnaire for User Interface Satisfaction. https://garyperlman.com/quest/quest.cgi?form=QUIS

S.O.S Emergencias. (2020). El Sistema S.O.S Emergencias. https://emergenciassos.com

Safe365. (2021). Safe 365. Cómo funciona. https://safe365.com/es/como-funciona

Seguros Bolívar. (2020). App Seguros Bolívar. https://www .appsegurosbolivar.com

Sura. (2017). App Seguros SURA. https://www.segurossura.com.co/landings/app/index.html

Unidad Nacional para la Gestión del Riesgo de Desastres. (2019). Consolidado anual de emergencias. http://portal.gestiondelriesgo.gov.co/Paginas/Consolidado-Atencion-de-Emergencias.aspx 